\title{
Existence Theory for the EED Inpainting Problem
}

\author{
Michael Bildhauer Marcelo Cárdenas Martin Fuchs \\ Joachim Weickert
}

Dedicated to Professor Nina Uraltseva on the occasion of her 85th birthday.

\begin{abstract}
We establish an existence theory for an elliptic boundary value problem in image analysis known as edge-enhancing diffusion (EED) inpainting. The EED inpainting problem aims at restoring missing data in an image as steady state of a nonlinear anisotropic diffusion process where the known data provide Dirichlet boundary conditions. We prove the existence of a weak solution by applying the LeraySchauder fixed point theorem and show that the set of all possible weak solutions is bounded. Moreover, we demonstrate that under certain conditions the sequences resulting from iterative application of the operator from the existence theory contain convergent subsequences.
\end{abstract}

AMS Subject Classification: 35J57, 94A08

Keywords: boundary value problems, anisotropic diffusion, Leray-Schauder fixed point theorem, inpainting, image restoration, image compression

\section{Introduction}

Restoring missing data in an EED inpainting problem means that we are given an open set $\Omega \subset \mathbb{R}^{2}$ as the area of an image and that the image data are just partially known, i.e. we consider a subset $K \subset \Omega$ and a function $f$ : $K \rightarrow[0,1]$. Here the values of $f$ represent the grey level between pure white and black.

As a typical example we may imagine that $K$ is a finite union of tiny regions like it is illustrated in Figure 1 below. 
The complete image is recovered by filling in the missing information at $G:=\Omega \backslash K$.

To this purpose, the boundary value problem

$$
\begin{aligned}
& \operatorname{div}\left(D\left(\nabla u_{\sigma}\right) \nabla u\right)=0 \text { on } G, \\
& u=f \quad \text { on } \partial K \text {, } \\
& D\left(\nabla u_{\sigma}\right) \nabla u \cdot \mathcal{N}=0 \quad \text { on } \quad \partial \Omega \backslash \partial K
\end{aligned}
$$

is studied, where $\mathcal{N}$ denotes the outward unit normal to $\Omega$ and where for any given function $w \in L^{1}(G)$ a Gaussian-smoothed version of $w$ with some fixed parameter $\sigma$ is denoted by $w_{\sigma}$.

In fact, this problem serves as a mathematical model for the anisotropic diffusion image inpainting problem [11, 13, 4].

The Dirichlet boundary condition (2) enforces the inpainted result $u$ to be coherent with the known data $f$ at the boundary of the known data region $K$. The problem is also supplemented with the natural Neumann boundary condition (3).

The diffusion tensor $D: \mathbb{R}^{2} \rightarrow \mathbb{R}^{2 \times 2}$ depending on the gradient of $u_{\sigma}$ is usually designed in order to steer the diffusion process in such a way that important geometrical information is taken into account.

Before going into details, we have to clarify our notation and assumptions regarding problem (1)-(3).

Notation. In the following we always suppose $\Omega \subset \mathbb{R}^{2}$ to be a bounded Lipschitz domain. For the definition of the Lebesgue- and Sobolev spaces $L^{p}(\Omega), W^{k, p}(\Omega)$ and its variants we refer to the monograph [1].

If $\sigma$ is a given positive parameter, then throughout this paper we denote for any function $w \in L^{1}(G)$ the globally smoothed function with the symbol $w_{\sigma}$,

$$
w_{\sigma}(x):=\int_{G} k_{\sigma}(x-z) w(z) \mathrm{d} z \quad \text { for all } \quad x \in \mathbb{R}^{2},
$$

where $k_{\sigma}$ denotes the Gaussian kernel

$$
k_{\sigma}(z):=\frac{1}{2 \pi \sigma^{2}} \exp \left(\frac{-|z|^{2}}{2 \sigma^{2}}\right) \quad \text { for all } \quad z \in \mathbb{R}^{2} .
$$

We use the symbol $c$ to denote a generic constant which may change from line to line. Whenever we want to stress its dependence on other values $\alpha_{1}, \alpha_{2}, \ldots$, we write $c\left(\alpha_{1}, \alpha_{2}, \ldots\right)$. 


\section{Main assumptions.}

i) We consider a finite number of open disjoint Lipschitz domains $C_{i} \subset$ $\Omega, C_{i} \neq \Omega, i=1, \ldots, N$, s.t. $\operatorname{dist}\left(C_{i}, C_{j}\right)>0$ for any $i \neq j, i$ $j \in\{1, \ldots, N\}$. We then define

$$
C:=\bigcup_{i=1}^{N} C_{i}, \quad K:=\bar{C}, \quad G:=\Omega \backslash K .
$$

For the sake of simplicity we assume throughout this paper that $K \Subset \Omega$, hence $\partial K \subset \partial G$. Otherwise we would have to decompose $\partial K=\Gamma_{1} \cup \Gamma_{2}$ with

$$
\Gamma_{1}:=\partial K \cap \partial G, \quad \Gamma_{2}=\partial K-\Gamma_{1} .
$$

ii) We suppose that the function $f: C \rightarrow[0,1]$ is of class $W^{1,2}(C)$. Extending this function to a function of class $W^{1,2}(\Omega)$, we use the same symbol $f$ with a slight abuse of notation.

iii) The diffusion tensor $D: \mathbb{R}^{2} \rightarrow \mathbb{R}^{2 \times 2}$ is assumed to be a continuous function satisfying the symmetry condition

$$
d_{\alpha, \beta}(p)=d_{\beta, \alpha}(p) \quad \text { with } \quad D(p)=\left(d_{\alpha, \beta}(p)\right)_{1 \leq \alpha \leq \beta \leq 2} \quad \text { for all } \quad p \in \mathbb{R}^{2} .
$$

Moreover, our hypothesis on non-degenerate ellipticity reads as: there exist positive constants $\lambda, c_{1}, c_{2}$ such that

$$
c_{1}\left(1+\frac{|p|^{2}}{\lambda^{2}}\right)^{-\frac{1}{2}}|q|^{2} \leq D(p) q \cdot q \leq c_{2}|q|^{2} \quad \text { for all } \quad p, q \in \mathbb{R}^{2} .
$$

EED inpainting. The choice of the ellipticity condition $(6)$ covers the inpainting problem with Edge-Enhancing Diffusion (EED) [11, 13, 4].

As an easy example, $D$ may look like (in fact its representation along the edge direction $\nabla u^{\perp}$ and across the edge direction, respectively, and the notion "edge direction" is motivated by the boundary of level sets of $u$ )

$$
D\left(\nabla u_{\sigma}\right):=\left(\begin{array}{cc}
1 & 0 \\
0 & \left(1+\left|\nabla u_{\sigma}\right|^{2}\right)^{-1 / 2}
\end{array}\right) .
$$

In other words, the diffusion tensor is such that the differential operator encourages diffusion along edges over diffusion across edges.

More precisely, $D\left(\nabla u_{\sigma}\right)$ is defined as a $2 \times 2$ matrix with eigenvectors $\nabla u_{\sigma}^{\perp}$ and $\nabla u_{\sigma}$ having as corresponding eigenvalues 1 and $g\left(\left|\nabla u_{\sigma}\right|^{2}\right)$, where $g$ is the 
Charbonnier diffusivity [3] given by $g(s):=\left(1+s^{2} / \lambda^{2}\right)^{-\frac{1}{2}}$.

Note that the inpainted image $u$ crucially depends on the steering parameter $\sigma$. Concerning this, a rigorous analysis remains an open problem.

However, for the rest of this work we may assume w.l.o.g. that $\lambda=1$ except when stated explicitly otherwise.

Edge-enhancing anisotropic diffusion was first proposed as a parabolic evolution process for denoising [11], where it can be regarded as an anisotropic alternative to isotropic regularisations [2] of the Perona-Malik filter [9]. This evolution has been shown to be well-posed in the continuous, space discrete, and fully discrete setting [12]. Later on, the elliptic steady state equation of EED has been supplemented with Dirichlet data and used for inpainting missing regions in matrix-valued images [13].

Its main application today is inpainting-based lossy image compression, where only a sparse, carefully optimised subset of the data is stored and the missing data are recovered by EED inpainting [4]. In this context, experiments have shown that EED gives state-of-the-art results that outperform other partial differential equations in terms of reconstruction quality [10].

Fig. 1 depicts an example for EED inpainting of sparse data, using a photo of Professor Nina Uraltseva. In spite of its qualities in practical applications, there is no existence theory for EED inpainting so far. Our paper closes this gap.

Main goal and summary. In the present work, we show the existence of a weak solution to problem (1)-(3) and present some properties of the set of possibly multiple weak solutions. Moreover, the canonical iteration procedure is analyzed by the way proving $W^{1,1}$-bounds in different settings.

Our paper is organized as follows: Section 2 covers the existence of a solution to (1)-(3). We define an operator $T: W^{1,1}(G) \rightarrow W^{1,1}(G)$ via an associated variational problem and the Leray-Schauder fixed point theorem [5, 6] gives a fixed point of $T$ and in turn the desired weak solution.

The analysis of the fixed point set outlined in Section 3 is motivated by the fact, that no results on the uniqueness of fixed points are available. Here we characterize the fixed points by another variational formulation.

The last section follows the idea, that suitable iterations of the operator $T$ may serve as a good approximation for a fixed point of $T$. 


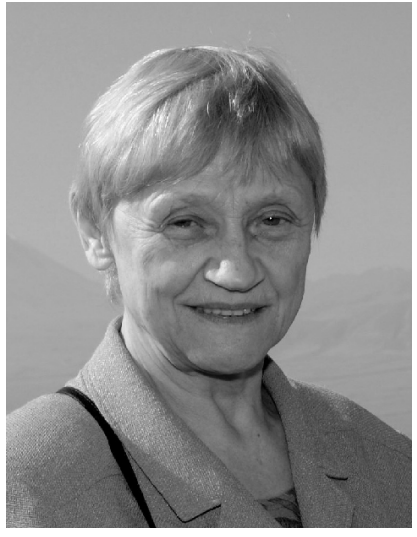

original image

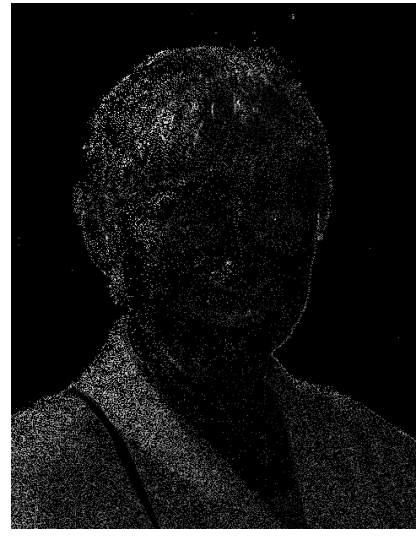

data kept $(10 \%)$

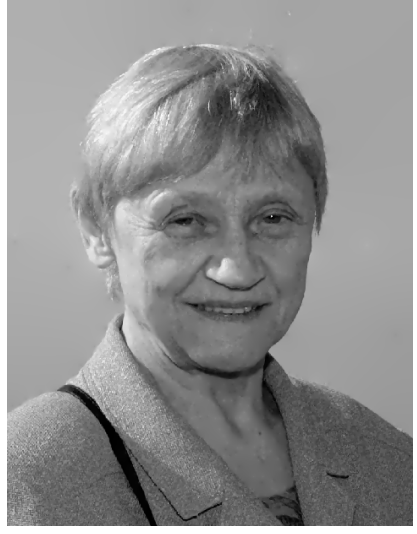

inpainted by EED

Figure 1: Illustration of EED inpainting. Left: Original image courtesy of Professor Uraltseva, $\Omega=(0,480) \times(0,623)$. Middle: Selecting $10 \%$ of the data with the probabilistic sparsification strategy from [7]. These data locations specify the set $K$. Right: Reconstruction from the sparsified data using a finite difference scheme for the EED inpainting model with $\lambda=1$ and $\sigma=0.8$.

For this kind of iteration, we shortly establish $W^{1,1}$-bounds in the case of sufficiently large $\sigma$ which in general fail to be true. Thus, uniform a priori estimates are based on a suitable smallness assumption.

Given these $W^{1,1}$-bounds, we are able to show the existence of fixed points w.r.t. an iterated operator $T^{n}$. We emphasize that these fixed points are found in a ball whose diameter just depends on the data and is independent of $\sigma$.

Concluding remarks deal with an interpretation of our error estimates.

\section{Existence theory}

The goal of this section is to show that under the above conditions problem (1)-(3) has at least one weak solution. Our strategy is to apply the LeraySchauder fixed point theorem:

First we define the weak solutions of the problem as the fixed points of an appropriate operator and then show that the operator has at least one fixed point [5, 6]. Note that $\sigma$ is fixed throughout this section.

Let us begin by introducing the relevant operator. For this purpose we need the following two ingredients: the class

$$
\mathcal{C}:=\left\{v \in W^{1,2}(G): v=f \text { on } \partial K \text { in the trace sense }\right\}
$$


and the family of functionals $J_{w}: W^{1,2}(G) \rightarrow \mathbb{R}$ defined as

$$
J_{w}(v):=\int_{G} D\left(\nabla w_{\sigma}\right) \nabla v \cdot \nabla v \mathrm{~d} x
$$

for any $w \in W^{1,1}(G)$ and any $v \in W^{1,2}(G)$.

Definition 2.1. The operator $T: W^{1,1}(G) \rightarrow W^{1,1}(G)$, is defined for any $w \in W^{1,1}(G)$ as the argumentum minimi (w.r.t. the class $\mathcal{C}$ )

$$
T(w):=\underset{v \in \mathcal{C}}{\arg \min } J_{w}(v) .
$$

For the sake of notational simplicity, the dependence on $\sigma$ is not highlighted unless we need a careful analysis proving uniform bounds in the last section.

This definition is well posed on account of Proposition 2.2, whose proof follows from the ellipticity of the diffusion tensor in a well known manner. We just recall the basic ingredients concerning Sobolev functions which in addition can be used as precise references throughout our whole exposition. The continuity of the trace operator follows, e.g., from Theorem 3.4.5 of [8], the variant of Poincaré's inequality from Theorem 3.6.4 of [8]

Proposition 2.1. Let $G \subset \mathbb{R}^{N}$ be a bounded open Lipschitz domain.

i) (Continuity of the trace operator)

For any $1 \leq p<\infty$ there exists a bounded linear operator $B: W^{1, p}(G) \rightarrow$ $L^{p}(\partial G)$ (the trace operator) such that $B(u)=\left.u\right|_{\partial G}$ whenever $u \in$ $W^{1, p}(G) \bigcap C(\bar{G})$. In particular, there exists a constant $c$, such that

$$
\|B u\|_{L^{p}(\partial G)} \leq c\|u\|_{W^{1, p}(G)},
$$

for any $u \in W^{1, p}(G)$.

ii) (Poincaré inequality)

Let $\Gamma_{1} \subset \partial G$ with $\mathcal{H}^{N-1}\left(\Gamma_{1}\right)>0$. If $u \in W^{1, p}(G)$ with $1 \leq p<\infty$ is such that $\left.u\right|_{\Gamma_{1}}=0$ in the trace sense, then

$$
\|u\|_{W^{1, p}(G)} \leq c\|\nabla u\|_{L^{p}(G)}
$$

for a positive constant $c$ which does not depend on $u$.

Proposition 2.2. The set $\mathcal{C}$ is a convex and weakly closed subset of $W^{1,2}(G)$. Moreover, for any fixed $w \in W^{1,1}(G)$, the functional $J_{w}$ is weakly lower semicontinuous over $\mathcal{C}$ and has a unique minimiser in $\mathcal{C}$. The unique minimiser $T(w)$ satisfies for all $v \in \mathcal{C}$

$$
\int_{G} D\left(\nabla w_{\sigma}\right) \nabla T(w) \cdot \nabla T(w) d x \leq \int_{G} D\left(\nabla w_{\sigma}\right) \nabla v \cdot \nabla v \mathrm{~d} x
$$

and for all $\phi \in W^{1,2}(G)$, such that $\left.\phi\right|_{\partial K}=0$ the Euler-Lagrange equation

$$
\int_{G} D\left(\nabla w_{\sigma}\right) \nabla T(w) \cdot \nabla \phi \mathrm{d} x=0 .
$$


Weak solution. Any fixed point of the operator $T$ is a weak solution of (1)-(3) in the following sense:

i) By definition, any fixed point $u$ of $T$ satisfies the Euler-Lagrange equation (12), hence $u$ is a weak solution of (1) in $G$.

ii) Any fixed point is of class $\mathcal{C}$, thus $(2)$ holds.

iii) Suppose that $x_{0} \in \partial \Omega$ and that there exists an open neighborhood $U \subset \mathbb{R}^{2}$ s.t. $u$ is globally of class $W^{2,2}$ on $U \cap \Omega$. Then we have (3) a.e. on $U \cap \partial \Omega$.

We are now ready to state our main result:

Theorem 2.1. (Existence of a weak solution) Suppose that we have our general hypotheses as stated above. Then the operator $T$ has at least one fixed point, i.e. the boundary value problem (1)-(3) is weakly solvable.

The proof of Theorem 2.1 is divided into different steps which we present as intermediate lemmas.

Lemma 2.1. Let $w \in W^{1,1}(G)$, then

$$
\|T(w)\|_{W^{1,1}(G)} \leq c\left(1+\|w\|_{L^{1}(G)}^{\frac{1}{2}}\right)
$$

for some positive constant $c$ which is independent of $w$.

Proof. First we estimate $\nabla w_{\sigma}$ in terms of $w$. It holds for any $x \in G$ that

$$
\partial_{\alpha} w_{\sigma}(x)=\int_{G} \partial_{\alpha} k_{\sigma}(x-y) w(y) \mathrm{d} y
$$

for $\alpha=1,2$. With 14 we find $c=c(G, \sigma)$ s.t.

$$
\left\|\nabla w_{\sigma}\right\|_{L^{\infty}(G)} \leq c\|w\|_{L^{1}(G)}
$$

and using the same argument, $(15)$ is established for all higher order derivatives.

Next we discuss the size of $\|T(w)\|_{W^{1,1}(G)}$. Fixing $w \in W^{1,1}(G)$, we have by Hölder's inequality that

$$
\begin{aligned}
& \int_{G}|\nabla T(w)| \mathrm{d} x \\
& =\int_{G}\left(1+\left|\nabla w_{\sigma}\right|^{2}\right)^{-\frac{1}{4}}|\nabla T(w)|\left(1+\left|\nabla w_{\sigma}\right|^{2}\right)^{\frac{1}{4}} \mathrm{~d} x \\
& \leq\left(\int_{G}\left(1+\left|\nabla w_{\sigma}\right|^{2}\right)^{-\frac{1}{2}}|\nabla T(w)|^{2} \mathrm{~d} x\right)^{\frac{1}{2}}\left(\int_{G}\left(1+\left|\nabla w_{\sigma}\right|^{2}\right)^{\frac{1}{2}} \mathrm{~d} x\right)^{\frac{1}{2}} \\
& \leq c\left(\int_{G}\left\langle D\left(\nabla w_{\sigma}\right) \nabla T(w), \nabla T(w)\right\rangle \mathrm{d} x\right)^{\frac{1}{2}}\left(\int_{G}\left(1+\left|\nabla w_{\sigma}\right|^{2}\right)^{\frac{1}{2}} \mathrm{~d} x\right)^{\frac{1}{2}}
\end{aligned}
$$


where for the last inequality we applied the ellipticity condition (6). Moreover, choosing $f$ as comparison function in (11) and applying (6) once again, we obtain

$$
A:=\int_{G} D\left(\nabla w_{\sigma}\right) \nabla T(w) \cdot \nabla T(w) \mathrm{d} x \leq c \int_{G}|\nabla f|^{2} \mathrm{~d} x
$$

whereas on account of 15, ,

$$
B:=\int_{G}\left(1+\left|\nabla w_{\sigma}\right|^{2}\right)^{\frac{1}{2}} \mathrm{~d} x \leq c\left(1+\|w\|_{L^{1}(G)}\right) .
$$

Applying these estimates of $A$ and $B$ to $(16)$, we obtain

$$
\|\nabla T(w)\|_{L^{1}(G)} \leq c A^{\frac{1}{2}} B^{\frac{1}{2}} \leq c\left(1+\|w\|_{L^{1}(G)}^{\frac{1}{2}}\right) .
$$

Since $T(w)$ is in the class $\mathcal{C}$, thus coinciding with $f$ over $\partial K \subset \partial G$, we may apply the Poincaré inequality (10) to the difference $T(w)-f$ and obtain

$$
\begin{aligned}
\|T(w)\|_{L^{1}(G)} & \leq\|f\|_{L^{1}(G)}+\|T(w)-f\|_{L^{1}(G)} \\
& \leq\|f\|_{L^{1}(G)}+c\|\nabla(T(w)-f)\|_{L^{1}(G)} \\
& \leq c\|f\|_{W^{1,1}(G)}+c\|\nabla T(w)\|_{L^{1}(G)} .
\end{aligned}
$$

Combining this estimate with inequality (17) we end up with 13 for a constant $c$ depending on the data $f, G$ and $\sigma$ but not depending on the function $w \in W^{1,1}(G)$ under consideration.

Lemma 2.2. The mapping $T: W^{1,1}(G) \rightarrow W^{1,1}(G)$ is continuous.

Proof. Let $w \in W^{1,1}(G)$ and $\left(w_{n}\right)_{n \in \mathbb{N}} \subset W^{1,1}(G)$ be such that

$$
\left\|w_{n}-w\right\|_{W^{1,1}(G)} \rightarrow 0 \quad \text { as } \quad n \rightarrow \infty .
$$

Moreover, let $u_{n}=T\left(w_{n}\right)$ and $u=T(w)$. We have to show that

$$
\left\|u_{n}-u\right\|_{W^{1,1}(G)} \rightarrow 0 \quad \text { as } \quad n \rightarrow \infty .
$$

In order to prove $(19)$, we begin with an observation concerning the mollifications: recalling (15) and (18) we have

$$
\nabla w_{\sigma}^{n} \rightarrow \nabla w_{\sigma} \quad \text { uniformly on } G \quad \text { as } \quad n \rightarrow \infty,
$$

where we use the symbol $w_{\sigma}^{n}$ instead of $\left(w_{n}\right)_{\sigma}$. The ellipticity condition (6), the continuity of the diffusion tensor and (20) imply for all $x \in G, q \in \mathbb{R}^{2}$ and for all $n \in \mathbb{N}$

$$
\mu_{1}|q|^{2} \leq D\left(\nabla w_{\sigma}^{n}(x)\right) q \cdot q \leq \mu_{2}|q|^{2}
$$


with constants $\mu_{1}, \mu_{2}>0$.

We again turn our attention to the sequence $u_{n}$ and combine the minimality of $u_{n}=T\left(w_{n}\right)$ (recall (11), use $f$ as admissible comparison function) with inequality (21) to obtain

$$
\mu_{1} \int_{G}\left|\nabla u_{n}\right|^{2} \mathrm{~d} x \leq \int_{G} D\left(\nabla w_{\sigma}^{n}\right) \nabla u_{n} \cdot \nabla u_{n} \mathrm{~d} x \leq \int_{G} D\left(\nabla w_{\sigma}^{n}\right) \nabla f \cdot \nabla f \mathrm{~d} x .
$$

Moreover, since $u_{n} \in \mathcal{C}$, we may apply Poincaré's inequality 10 in order to get the estimate

$$
\left\|u_{n}\right\|_{L^{2}(G)} \leq\left\|u_{n}-f\right\|_{L^{2}(G)}+\|f\|_{L^{2}(G)} \leq c\left(1+\left\|\nabla u_{n}\right\|_{L^{2}(G)}\right)
$$

again with a constant just depending on the data. Inequalities $(22)$ and $(23)$ are put together with the following result of the first step:

$$
\sup _{n \in \mathbb{N}}\left\|u_{n}\right\|_{W^{1,2}(G)}<\infty
$$

Then, by the Banach-Alaoglu theorem, there exists a subsequence $\left(u_{n_{k}}\right)_{k \in \mathbb{N}}$ of $\left(u_{n}\right)_{n \in \mathbb{N}}$ s.t. for some $\tilde{u} \in W^{1,2}(G)$

$$
u_{n_{k}} \rightarrow \tilde{u} \quad \text { in } \quad W^{1,2}(G) \text { as } \quad k \rightarrow \infty .
$$

In the next step we show that in fact $\tilde{u}=u$ which immediately gives the convergence of the sequence as a whole:

$$
u_{n} \rightarrow u \text { in } W^{1,2}(G) \text { as } n \rightarrow \infty .
$$

In fact, since $\mathcal{C}$ is a closed convex subset of $W^{1,2}(G)$, Mazur's lemma implies that it is also weakly closed, hence $\tilde{u} \in \mathcal{C}$.

On the other hand, we have 12 w.r.t. the minimisers $u_{n_{k}}=T\left(w_{n_{k}}\right)$ : it holds that

$$
\int_{G} D\left(\nabla w_{\sigma}^{n_{k}}\right) \nabla u_{n_{k}} \cdot \nabla \phi \mathrm{d} x=0
$$

for any $\phi \in W^{1,2}(G)$ satisfying $\left.\phi\right|_{\partial K}=0$. Hence, applying (20) and 25 in equation (27) we arrive at the limit (w.r.t. $n_{k}$ ) equation

$$
\int_{G} D\left(\nabla w_{\sigma}\right) \nabla \tilde{u} \cdot \nabla \phi \mathrm{d} x=0 \text { for any } \phi \text { as above. }
$$

By definition $u$ is a solution of (28) as well which, by uniqueness, is just possible provided that $\tilde{u}=u$ and we have established our claim (26).

It remains to improve 26 towards

$$
\nabla u_{n} \rightarrow \nabla u \quad \text { in } \quad L^{2}\left(G, \mathbb{R}^{2}\right) \quad \text { as } \quad n \rightarrow \infty
$$


which gives (19) as a byproduct. We have the Euler-Lagrange equations (12) both for $u=T(w)$ and for $u_{n}=T\left(w_{n}\right)$ with $\phi:=u_{n}-u$ as admissible test function

$$
\int_{G} D\left(\nabla w_{\sigma}\right) \nabla u \cdot\left(\nabla u_{n}-\nabla u\right) \mathrm{d} x=\int_{G} D\left(\nabla w_{\sigma}^{n}\right) \nabla u_{n} \cdot\left(\nabla u_{n}-\nabla u\right) \mathrm{d} x=0,
$$

which, together with the ellipticity (6), shows for some $c>0$

$$
\begin{aligned}
c \int_{G}\left|\nabla u_{n}-\nabla u\right|^{2} \mathrm{~d} x & \leq \int_{G} D\left(\nabla w_{\sigma}\right)\left(\nabla u_{n}-\nabla u\right) \cdot\left(\nabla u_{n}-\nabla u\right) \mathrm{d} x \\
& =\int_{G}\left(D\left(\nabla w_{\sigma}\right)-D\left(\nabla w_{\sigma}^{n}\right)\right) \nabla u_{n} \cdot\left(\nabla u_{n}-\nabla u\right) \mathrm{d} x .
\end{aligned}
$$

We finally observe that $D\left(\nabla w_{\sigma}^{n}\right) \rightarrow D\left(\nabla w_{\sigma}\right)$ uniformly on $G$ (by the continuity of $D$ and by (20)) which, together with the uniform bound (24), gives (29) and the proof is completed.

The last auxiliary lemma reads as:

Lemma 2.3. The continuous mapping $T: W^{1,1}(G) \rightarrow W^{1,1}(G)$ is compact.

Proof. Suppose that we are given a sequence $\left(w_{n}\right)_{n \in \mathbb{N}} \subset W^{1,1}(G)$ s.t.

$$
\sup _{n \in \mathbb{N}}\left\|w_{n}\right\|_{W^{1,1}(G)}<\infty .
$$

Letting $u_{n}:=T\left(w_{n}\right)$ we then have to extract a convergent subsequence $\left(u_{n_{k}}\right)_{k \in \mathbb{N}}$,

$$
u_{n_{k}} \rightarrow: \hat{u} \quad \text { in } W^{1,1}(G) \text { as } k \rightarrow \infty,
$$

where we do not claim that $\hat{u}$ is a solution of some limit equation.

An account of our hypothesis (30) we can exactly follow the lines outlined in the proof of Lemma 2.2 and reproduce (24) in the situation at hand. Passing to a subsequence we may suppose

$$
u_{n_{k}} \rightarrow: \hat{u} \quad \text { in } W^{1,2}(G), \quad u_{n_{k}} \rightarrow \hat{u} \quad \text { in } L^{s}(G) \text { for any } 1<s<\infty .
$$

We finally establsh $L^{2}$-convergence of the gradients, which in particular proves the claim (31).

In fact, observe that on account of (15) and (30) the functions

$$
A_{n_{k}}: \bar{G} \rightarrow \mathbb{R}^{2 \times 2}, \quad A_{n_{k}}(x):=D\left(\nabla w_{\sigma}^{n_{k}}(x)\right),
$$

are bounded and equicontinuous on $\bar{G}$. Thus by Arzela's theorem there exist a further subsequence, with a slight abuse of notation still denoted by $\left(A_{n_{k}}\right)_{k \in \mathbb{N}}$, and a function $A: \bar{G} \rightarrow \mathbb{R}^{2 \times 2}$ s.t. as $k \rightarrow \infty$

$$
A_{n_{k}} \rightrightarrows A \text { uniformly in } \bar{G} \text {. }
$$


We write

$$
\begin{array}{rl}
\int_{G} & A(x)\left(\nabla u_{n_{k}}-\nabla \hat{u}\right) \cdot\left(\nabla u_{n_{k}}-\nabla \hat{u}\right) \mathrm{d} x \\
= & \int_{G} A_{n_{k}}(x)\left(\nabla u_{n_{k}}-\nabla \hat{u}\right) \cdot\left(\nabla u_{n_{k}}-\nabla \hat{u}\right) \mathrm{d} x \\
\quad & \quad+\int_{G}\left(A(x)-A_{n_{k}}(x)\right)\left(\nabla u_{n_{k}}-\nabla \hat{u}\right) \cdot\left(\nabla u_{n_{k}}-\nabla \hat{u}\right) \mathrm{d} x \\
= & \quad \alpha_{k}+\beta_{k} .
\end{array}
$$

The uniform convergence $A_{n_{k}} \rightrightarrows A$ implies (recalling (24))

$$
\lim _{k \rightarrow \infty} \beta_{k}=0 \quad \text { as } \quad k \rightarrow \infty \text {. }
$$

As in the proof of Lemma 2.2, the Euler-Lagrange equation w.r.t. $u_{n_{k}}=$ $T\left(w_{n_{k}}\right)$ is applied leading to

$$
\begin{aligned}
\alpha_{k}= & -\int_{G} A_{n_{k}}(x) \nabla \hat{u} \cdot\left(\nabla u_{n_{k}}-\nabla \hat{u}\right) \mathrm{d} x \\
= & \int_{G}\left(A(x)-A_{n_{k}}(x)\right) \nabla \hat{u} \cdot\left(\nabla u_{n_{k}}-\nabla \hat{u}\right) \mathrm{d} x \\
& -\int_{G} A(x) \nabla \hat{u} \cdot\left(\nabla u_{n_{k}}-\nabla \hat{u}\right) \mathrm{d} x .
\end{aligned}
$$

Here the second term is handled with the weak convergence of $u_{n_{k}}$ (see $(32)$ ), for the first one we argue in addition with the uniform convergence $A_{n_{k}} \rightrightarrows A$ to obtain

$$
\lim _{k \rightarrow \infty} \alpha_{k}=0 \quad \text { as } \quad k \rightarrow \infty
$$

Summarizing the results, (33), (34) and (35) yield the main equality

$$
\lim _{k \rightarrow \infty} \int_{G} A(x)\left(\nabla u_{n_{k}}-\nabla \tilde{u}\right) \cdot\left(\nabla u_{n_{k}}-\nabla \tilde{u}\right) \mathrm{d} x=0 .
$$

As a final remark we just mention that the condition

$$
\mu_{1}|q|^{2} \leq A(x) q \cdot q
$$

for all $x \in G, q \in \mathbb{R}^{2}$ and for some positive constant $\mu_{1}$ is induced by the uniform convergence, which completes the proof.

Proof of Theorem 2.1. The theorem is proved once we have verified the assumptions of [5], Theorem 11.3. 
By Lemma 2.3, $T$ is compact mapping and it remains to prove the existence of a positive constant $M$ with the property

$$
\|u\|_{W^{1,1}(G)}<M \quad \text { for all } u \in W^{1,1}(G) \text { and for all } \alpha \in[0,1] \text { s.t. } u=\alpha T(u) .
$$

Lemma 2.1 in particular shows

$$
\|T(u)\|_{W^{1,1}(G)} \leq c\left(1+\|u\|_{W^{1,1}(G)}^{\frac{1}{2}}\right) .
$$

W.l.o.g. we may assume $\alpha>0$. If $u=\alpha T(u)$, then

$$
\|u\|_{W^{1,1}(G)} \leq \alpha c\left(1+\|u\|_{W^{1,1}(G)}^{\frac{1}{2}}\right)
$$

and using Young's inequality, we obtain for any $\epsilon>0$

$$
\left(1-\frac{\epsilon \alpha c}{2}\right)\|u\|_{W^{1,1}(G)} \leq \alpha c\left(1+\frac{1}{2 \epsilon}\right) .
$$

By elementary calculations we may choose

$$
M=\alpha c \frac{1+2 \epsilon}{\epsilon(2-\epsilon \alpha c)}
$$

letting in addition $\epsilon=1 / c$ and the claim follows.

\section{Analysis of the fixed point set}

In the previous section we proved the existence of at least one solution to (1)-(3) using the Leray-Schauder fixed point argument.

Since no information on the uniqueness of solutions is available, the analysis of the fixed point set is of particular interest. In this section we study the properties of the set of possibly multiple weak solutions.

We still fix a smoothing parameter $\sigma>0$ throughout this section.

In Proposition 3.1 we show that the set

$$
\mathcal{F}:=\left\{u \in W^{1,1}(G): u=T(u)\right\}
$$

of fixed points of $T$ is bounded.

Then, in Proposition 3.2, we introduce an appropriate functional $\mathcal{J}$ and characterize the set $\mathcal{F}$ as the set of all weak $W^{1,2}$-limits of $\mathcal{J}$-minimizing sequences. 
Proposition 3.1. The set $\mathcal{F}$ is a bounded subset of $W^{1,2}(G)$.

Proof. By definition we have $T\left(W^{1,1}(G)\right) \subset \mathcal{C}$ (recall (7)). Thus, Poincaré's inequality (10) can be applied to any function $w \in \mathcal{F}$ and we find a constant $c=c(\sigma, f, G)$ s.t.

$$
\begin{aligned}
\|w\|_{L^{1}(G)} & \leq\|w-f\|_{L^{1}(G)}+\|f\|_{L^{1}(G)} \\
& \leq c\left(1+\|\nabla w\|_{L^{1}(G)}\right) .
\end{aligned}
$$

Lemma 2.1 yields for $w=T(w)$

$$
\|\nabla w\|_{L^{1}(G)}=\|\nabla T(w)\|_{L^{1}(G)} \leq c\left(1+\|\nabla w\|_{L^{1}(G)}^{\frac{1}{2}}\right) .
$$

Youngs' inequality then immediately gives

$$
\|\nabla w\|_{L^{1}(G)} \leq c(\sigma, f, G)=: \kappa<\infty \text { for all } w \in \mathcal{F} .
$$

Note that (37) implies as above

$$
\|w\|_{L^{1}(G)} \leq c(\kappa) .
$$

Finally we may apply (15) once again together with the ellipticity condition (6), which proves the proposition on account of

$$
\int_{G}|\nabla w|^{2} \mathrm{~d} x \leq c(\kappa) \int_{G} D\left(\nabla w_{\sigma}(x)\right) \nabla w \cdot \nabla w \mathrm{~d} x \leq c(\kappa)\|\nabla f\|_{L^{2}(G)}^{2},
$$

where the last inequality follows from the minimality of $w=T(w)$.

Next we consider the functional

$$
\mathcal{J}: \mathcal{C} \rightarrow \mathbb{R}, \quad \mathcal{J}[u]:=\int_{G}|\nabla u-\nabla T(u)|^{2} \mathrm{~d} x
$$

and the set of cluster points

$$
\begin{aligned}
\mathcal{M}:= & \left\{u \in W^{1,2}(G):\right. \\
& \left.u \text { is the weak } W^{1,2} \text {-limit of a } \mathcal{J} \text {-minimizing sequence }\right\} .
\end{aligned}
$$

Proposition 3.2. The sets $\mathcal{F}$ and $\mathcal{M}$ coincide, i.e. $\mathcal{F}=\mathcal{M}$.

Proof. The inclusion $\mathcal{F} \subset \mathcal{M}$ follows (considering a fixed point as a contant sequence) from Proposition 3.1 which gives $\mathcal{F} \subset W^{1,2}(G)$. Moreover, we observe that the existence of a fixed point (Theorem 2.1) implies

$$
\inf _{\mathcal{C}} \mathcal{J}=0 .
$$


Now fix any $\mathcal{J}$-minimizing sequence $\left(u_{n}\right)_{n \in \mathbb{N}} \subset \mathcal{C}$ with weak $W^{1,2}$-cluster point $u$, i.e. we suppose after passing to a subsequence (not relabeled)

$$
u_{n} \rightarrow: \hat{u} \quad \text { in } W^{1,2}(G)
$$

and claim that $\hat{u}$ is a fixed point of $T$, i.e. $\hat{u}=T(\hat{u})$. Note that $\mathcal{C}$ is weakly closed, i.e. $\hat{u} \in \mathcal{C}$.

We have

$$
\sup _{n \in \mathbb{N}}\left\|u_{n}\right\|_{W^{1,2}(G)}<\infty
$$

and by the $\mathcal{J}$-minimizing property of the sequence

$$
\sup _{n \in \mathbb{N}}\left\|T\left(u_{n}\right)\right\|_{W^{1,2}(G)}<\infty .
$$

From 43 and 40 we a find subsequence $\left(u_{n_{k}}\right)_{k \in \mathbb{N}} \subset\left(u_{n}\right)_{n \in \mathbb{N}}$ s.t.

$$
u_{n_{k}} \rightarrow: \hat{u}, \quad T\left(u_{n_{k}}\right) \rightarrow: \xi \quad \text { in } W^{1,2}(G) .
$$

where we also have $\xi \in \mathcal{C}$. Moreover, by 15 and Arzela's theorem, we may assume the uniform convergence on $G$ as $k \rightarrow \infty$ :

$$
D\left(\nabla u_{\sigma}^{n_{k}}\right) \rightrightarrows D\left(\nabla \hat{u}_{\sigma}\right)
$$

Arguing with lower semicontinuity (see (41)), the uniform convergence (42), the minimality of $T\left(u_{n_{k}}\right)$ and once again with 42 we have for any $v \in \mathcal{C}$

$$
\begin{aligned}
\int_{G} D\left(\nabla \hat{u}_{\sigma}\right) \nabla \xi \cdot \nabla \xi \mathrm{d} x & \leq \liminf _{k \rightarrow \infty} \int_{G} D\left(\nabla \hat{u}_{\sigma}\right) \nabla T\left(u_{n_{k}}\right) \cdot \nabla T\left(u_{n_{k}}\right) \mathrm{d} x \\
& \leq \liminf _{k \rightarrow \infty} \int_{G} D\left(\nabla u_{\sigma}^{n_{k}}\right) \nabla T\left(u_{n_{k}}\right) \cdot \nabla T\left(u_{n_{k}}\right) \mathrm{d} x \\
& \leq \liminf _{n \rightarrow \infty} \int_{G} D\left(\nabla u_{\sigma}^{n_{k}}\right) \nabla v \cdot \nabla v \mathrm{~d} x \\
& \leq \int_{G} D\left(\nabla \hat{u}_{\sigma}\right) \nabla v \cdot \nabla v \mathrm{~d} x
\end{aligned}
$$

This minimizing property of $\xi$ implies by the uniqueness of solutions $\xi=$ $T(\hat{u})$. Finally pointwise a.e. convergence of $u_{n_{k}}$ and $T\left(u_{n_{k}}\right)$ show the claim $\hat{u}=T(\hat{u})$.

Remark 3.1. In fact, any $\mathcal{J}$-minimizing sequence $\left(u_{n}\right)_{n \in \mathbb{N}}$ satisfies

$$
\sup _{n \in \mathbb{N}}\left\|u_{n}\right\|_{W^{1,2}(G)}<\infty
$$

which guarantees the existence of weakly convergent subsequences. 
Proof. Fix a $\mathcal{J}$-minimizing sequence $\left(u_{n}\right)_{n \in \mathbb{N}}$. The inequality

$$
\left\|\nabla u_{n}\right\|_{L^{1}(G)} \leq\left\|\nabla u_{n}-\nabla T\left(u_{n}\right)\right\|_{L^{1}(G)}+\left\|\nabla T\left(u_{n}\right)\right\|_{L^{1}(G)}
$$

together with (recall (38))

$$
\left\|\nabla u_{n}-\nabla T\left(u_{n}\right)\right\|_{L^{1}(G)} \leq|G|^{\frac{1}{2}} \mathcal{J}\left[u_{n}\right]^{\frac{1}{2}} \rightarrow 0 \quad \text { as } n \rightarrow \infty
$$

yields for any $\epsilon>0$

$$
\left\|\nabla u_{n}\right\|_{L^{1}(G)} \leq\left\|\nabla T\left(u_{n}\right)\right\|_{L^{1}(G)}+\epsilon
$$

provided that $n$ is sufficiently large. Exactly as outlined in the proof of Proposition 3.1 we derive

$$
\left\|\nabla T\left(u_{n}\right)\right\|_{L^{1}(G)} \leq c\left(1+\left\|\nabla u_{n}\right\|_{L^{1}(G)}^{\frac{1}{2}}\right),
$$

and with Young's inequality we get

$$
\sup _{n \in \mathbb{N}}\left\|u_{n}\right\|_{W^{1,1}(G)}<\infty .
$$

On account of 43 we now may follow the concluding remarks proving Proposition 3.1 with the result

$$
\left\|\nabla T\left(u_{n}\right)\right\|_{L^{2}(G)}^{2} \leq \int_{G} D\left(\nabla u_{\sigma}^{n}\right) \nabla T\left(u_{n}\right) \cdot \nabla T\left(u_{n}\right) \mathrm{d} x \leq c,
$$

hence

$$
\sup _{n \in \mathbb{N}}\left\|T\left(u_{n}\right)\right\|_{W^{1,2}(G)}<\infty .
$$

Since the sequence is $J$-minimizing, Remark 3.1 is obvious.

\section{$4 \quad$ Study of iterated sequences}

In this section we are interested in the question, whether the above considerations provide a rigorous analytical framework for an iteration of the operator $T$ as approximation of the EED inpainting problem under consideration.

More precisely, recalling (8) we fix $u^{(0)} \in W^{1,1}(G)$ s.t. $u^{(0)}=f$ on $\partial K$ and let

$$
u^{(j+1)}:=T\left(u^{(j)}\right), \quad j \in \mathbb{N}_{0} .
$$

Here and in the following the dependence of the operator $T$ on the parameter $\sigma$ is of particular interest. Nevertheless we keep the notation of the previous sections and do not highlight the dependence on $\sigma$. 
In Section 4.1 it becomes evident, that for arbitrary values of $\sigma$ we even cannot expect uniform $W^{1,1}$-estimates for an iteration of $T$. We just shortly sketch that assuming $\sigma$ to be large enough leads to $W^{1,1}$-bounds without further constraints on the data.

In Section 4.2 some refined a priori estimates are presented which lead to fixed points of the iterated operator $T^{n}$ in a priori bounded balls $\mathcal{U} \subset W^{1,1}(G)$. However, these estimates rely on a suitable smallness condition on the data.

We finish this section with some concluding remark on error estimates.

\subsection{Iterated sequences with large $\sigma$}

Proposition 4.1. Consider $u^{(j)}$ as given in (44). There exists a constant $\rho>0$ (compare (48)) s.t. in case $\sigma^{4}>\rho$

$$
\sup _{j \in \mathbb{N}_{0}}\left\|u^{(j)}\right\|_{W^{1,1}(G)}<\infty
$$

Proof. For any $w \in \mathcal{C}$ we have $\nabla w_{\sigma}(x)=\int_{G} \nabla_{x} k_{\sigma}(x-z) w(z) \mathrm{d} z$, hence by the definition off the Gaussian kernel

$$
\left|\nabla w_{\sigma}(x)\right| \leq \frac{1}{2 \pi \sigma^{4}}\|w\|_{L^{1}(G)} .
$$

Let us recall Poincaré's inequality 10 by writing $c=c_{P}$,

$$
\|w\|_{L^{1}(G)} \leq c_{P}\left(\|\nabla(w-f)\|_{L^{1}(G)}+\|f\|_{L^{1}(G)}\right),
$$

Specifying the constants occuring in (16) and recalling the uniform ellipticity (6) we obtain

$$
\|\nabla T(w)\|_{L^{1}(G)} \leq 2\left(\frac{c_{2}}{c_{1}}\right)^{\frac{1}{2}}\|\nabla f\|_{L^{2}(G)}\left(1+\left\|\nabla w_{\sigma}\right\|_{L^{1}(G)}\right) .
$$

Now let

$$
\begin{aligned}
\rho_{1} & :=2\left(\frac{c_{2}}{c_{1}}\right)^{\frac{1}{2}}\|\nabla f\|_{L^{2}(G)}\left[1+\frac{|G|}{2 \pi \sigma^{4}} c_{P}\left(\|f\|_{L^{1}(G)}+\|\nabla f\|_{L^{1}(G)}\right)\right], \\
\rho_{2} & :=2\left(\frac{c_{2}}{c_{1}}\right)^{\frac{1}{2}}\|\nabla f\|_{L^{2}(G)} \frac{|G|}{2 \pi} c_{P}, \\
\rho & :=\max \left\{\rho_{1}, \rho_{2}\right\},
\end{aligned}
$$

thus 45, 46 and 47) yield

$$
\|\nabla T(w)\|_{L^{1}(G)} \leq \rho\left(1+\sigma^{-4}\|\nabla w\|_{L^{1}(G)}\right) .
$$


Applying iteratively 49 to $u^{(j)}$ we obtain that

$$
\left\|\nabla u^{(j)}\right\|_{L^{1}(G)} \leq \sum_{k=1}^{\infty} \rho^{k} \sigma^{-4(k-1)}+\rho^{j} \sigma^{-4 j}\left\|\nabla u^{(0)}\right\|_{L^{1}(G)} .
$$

Hence, if we assume $\sigma^{4}>\rho$, then a final application of Poincaré's inequality shows the proposition.

Using the compactness of the operator $T$ and the same reasoning as in Lemma 2.2 we immediately obtain as a byproduct

Corollary 4.1. Consider $u^{(j)}$ as given in (44) and suppose that $\sigma^{4}>\rho$ with $\rho$ given in (48).

i) There exists a subsequence $\left(u^{\left(j_{k}\right)}\right)_{k \in \mathbb{N}} \subset\left(u^{(j)}\right)_{n \in \mathbb{N}_{0}}$ and a function $u \in$ $W^{1,1}(G)$ such that $u^{\left(j_{k}\right)} \rightarrow u \in W^{1,1}(G)$ as $k \rightarrow \infty$.

ii) We have

$$
\sup _{j \in \mathbb{N}_{0}}\left\|u^{(j)}\right\|_{W^{1,2}(G)}<\infty
$$

\subsection{Iterated sequences with small $\sigma$}

Throughout this section, $\sigma$ is some arbitrary (small) fixed positive real number. In particular, $\sigma$ is not bounded from below by the data of the problem.

\subsubsection{A priori estimates}

Here we derive the main tool for the analysis of the sequence $\left\{u^{(j)}\right\}$, where we recall the definition (44) of this sequence and once more emphasize the ellipticity condition (6), where $\lambda>0$ now is handled as a free parameter.

Similar to 16 one observes for $w \in W^{1,1}(G), w=f$ on $\partial K$,

$$
\begin{aligned}
\| & \nabla T(w) \|_{L^{1}(G)} \\
\leq & \int_{G}\left(\frac{\left(c_{1} \lambda\right)^{\frac{1}{2}}}{\left(\lambda^{2}+\left|\nabla w_{\sigma}\right|^{2}\right)^{\frac{1}{4}}}|\nabla T(w)| \cdot \frac{\left(\lambda^{2}+\left|\nabla w_{\delta}\right|^{2}\right)^{\frac{1}{4}}}{\left(c_{1} \lambda\right)^{\frac{1}{2}}}\right) \mathrm{d} x \\
& \leq\left(\int_{G} \frac{c_{1} \lambda}{\left(\lambda^{2}+\left|\nabla w_{\sigma}\right|^{2}\right)^{\frac{1}{2}}}|\nabla T(w)|^{2} \mathrm{~d} x\right)^{\frac{1}{2}}\left(\int_{G} \frac{1}{c_{1} \lambda}\left(\lambda^{2}+\left|\nabla w_{\sigma}\right|^{2}\right)^{\frac{1}{2}} \mathrm{~d} x\right)^{\frac{1}{2}} \\
=: & A^{\frac{1}{2}} B^{\frac{1}{2}} .
\end{aligned}
$$


Condition (6) and the minimality of $T(w)$ give as before

$$
A^{\frac{1}{2}} \leq c_{2}^{\frac{1}{2}}\left(\int_{G}|\nabla f|^{2} \mathrm{~d} x\right)^{\frac{1}{2}}
$$

Using the elementary inequality

$$
(a+b)^{\frac{1}{2}} \leq a^{\frac{1}{2}}+b^{\frac{1}{2}} \quad \text { for all } a, b \geq 0
$$

we further obtain (now emphasizing the precise constants)

$$
\begin{aligned}
B^{\frac{1}{2}} & =\frac{1}{\left(c_{1} \lambda\right)^{\frac{1}{2}}}\left(\int_{G}\left(\lambda^{2}+\left|\nabla w_{\sigma}\right|^{2}\right)^{\frac{1}{2}} \mathrm{~d} x\right)^{\frac{1}{2}} \leq \frac{1}{\left(c_{1} \lambda\right)^{\frac{1}{2}}}\left(\int_{G}\left(\lambda+\left|\nabla w_{\sigma}\right|\right) \mathrm{d} x\right)^{\frac{1}{2}} \\
& \leq \frac{1}{\left(c_{1} \lambda\right)^{\frac{1}{2}}}\left(\lambda|G|+\left\|\nabla w_{\sigma}\right\|_{L^{1}(G)}\right)^{\frac{1}{2}} \leq \frac{1}{\left(c_{1} \lambda\right)^{\frac{1}{2}}}\left(\lambda^{\frac{1}{2}}|G|^{\frac{1}{2}}+\left\|\nabla w_{\sigma}\right\|_{L^{1}(G)}^{\frac{1}{2}}\right) .
\end{aligned}
$$

Thus, it is shown that for all $w \in W^{1,1}(G), w=f$ on $\partial K$,

$$
\|\nabla T(w)\|_{L^{1}(G)} \leq c_{2}^{\frac{1}{2}}\left(\int_{G}|\nabla f|^{2} \mathrm{~d} x\right)^{\frac{1}{2}} \frac{1}{\left(c_{1} \lambda\right)^{\frac{1}{2}}}\left(\lambda^{\frac{1}{2}}|G|^{\frac{1}{2}}+\left\|\nabla w_{\sigma}\right\|_{L^{1}(G)}^{\frac{1}{2}}\right) .
$$

Note that, for instance, the constant occurring in (15) strongly depends on $\sigma$, thus we now proceed in a different manner using an integration by parts

$$
\begin{aligned}
\left\|\nabla w_{\sigma}\right\|_{L^{1}(G)} & =\left|\int_{G} \int_{G} \nabla_{x} k_{\sigma}(x-z) w(z) \mathrm{d} z \mathrm{~d} x\right| \\
& \leq\left|\int_{G} \int_{G} \nabla_{z} k_{\sigma}(x-z) w(z) \mathrm{d} z \mathrm{~d} x\right| \\
& \leq\left\|(\nabla w)_{\sigma}\right\|_{L^{1}(G)}+\|w\|_{L^{1}(\partial G)} .
\end{aligned}
$$

Next, the well known property

$$
\left\|(\nabla w)_{\sigma}\right\|_{L^{1}(G)}=\int_{G}\left|\int_{G} k_{\sigma}(x-z) \nabla w(z) \mathrm{d} z\right| \mathrm{d} x \leq\|\nabla w\|_{L^{1}(G)}
$$

is used. Denoting the constant in the trace inequality (9) by $c_{T}$, the second term on the r.h.s. of (52) is handled via (recalling Poincaré's inequality)

$$
\begin{aligned}
\|w\|_{L^{1}(\partial G)} & \leq c_{T}\|w\|_{W^{1,1}(G)} \\
& \leq c_{T}\left[\|w-f\|_{W^{1,1}(G)}+\|f\|_{W^{1,1}(G)}\right] \\
& \leq c_{T}\left[c_{P}\|\nabla w\|_{L^{1}(G)}+\left(1+c_{P}\right)\|f\|_{W^{1,1}(G)}\right] .
\end{aligned}
$$


Altogether, (52), (53) and (54) imply

$$
\left\|\nabla w_{\sigma}\right\|_{L^{1}(G)} \leq\left(1+c_{T} c_{P}\right)\|\nabla w\|_{L^{1}(G)}+c_{T}\left(1+c_{P}\right)\|f\|_{W^{1,1}(G)} .
$$

Thus, (51) and 55) yield

$$
\begin{aligned}
\|\nabla T(w)\|_{L^{1}(G)} \leq & c_{2}^{\frac{1}{2}}\left(\int_{G}|\nabla f|^{2} \mathrm{~d} x\right)^{\frac{1}{2}} \\
& \cdot \frac{1}{\left(c_{1} \lambda\right)^{\frac{1}{2}}}\left[\lambda^{\frac{1}{2}}|G|^{\frac{1}{2}}+\left(1+c_{T} c_{P}\right)^{\frac{1}{2}}\|\nabla w\|_{L^{1}(G)}^{\frac{1}{2}}\right. \\
& \left.+c_{T}^{\frac{1}{2}}\left(1+c_{P}\right)^{\frac{1}{2}}\|f\|_{W^{1,1}(G)}^{\frac{1}{2}}\right] .
\end{aligned}
$$

Define the constants

$$
\begin{aligned}
& K_{1}:=\left(\frac{c_{2}}{c_{1}}\right)^{\frac{1}{2}}\|\nabla f\|_{L^{2}(G)}\left(|G|^{\frac{1}{2}}+\frac{c_{T}^{\frac{1}{2}}}{\lambda^{\frac{1}{2}}}\left(1+c_{P}\right)^{\frac{1}{2}}\|f\|_{W^{1,1}(G)}^{\frac{1}{2}}\right), \\
& K_{2}:=\frac{1}{\lambda^{\frac{1}{2}}}\left(\frac{c_{2}}{c_{1}}\right)^{\frac{1}{2}}\|\nabla f\|_{L^{2}(G)}\left(1+c_{T} c_{P}\right)^{\frac{1}{2}}
\end{aligned}
$$

and reformulate (56) using (57) and (58) as

$$
\|\nabla T(w)\|_{L^{1}(G)} \leq K_{1}+K_{2}\|\nabla w\|_{L^{1}(G)}^{\frac{1}{2}},
$$

which allows us to prove:

Theorem 4.1. With the notation of above we have for all $j \geq 2$

$$
\left\|\nabla u^{(j)}\right\|_{L^{1}(G)} \leq \sum_{l=1}^{j} K_{1}^{\frac{1}{2^{l-1}}} K_{2}^{\sum_{i=0}^{l-2} \frac{1}{2^{i}}}+K_{2}^{\sum_{i=0}^{j-1} \frac{1}{2^{i}}}\left\|\nabla u^{(0)}\right\|_{L^{1}(G)}^{\frac{1}{2^{j}}} .
$$

Proof by induction. For $j=2$ inequality (59) implies

$$
\begin{aligned}
\left\|\nabla u^{(2)}\right\|_{L^{1}(G)} & =\left\|\nabla T\left(u^{(1)}\right)\right\|_{L^{1}(G)} \\
& \leq K_{1}+K_{2}\left\|\nabla u^{(1)}\right\|_{L^{1}(G)}^{\frac{1}{2}} \\
& \leq K_{1}+K_{2}\left[K_{1}+K_{2}\left\|\nabla u^{(0)}\right\|_{L^{1}(G)}^{\frac{1}{2}}\right]^{\frac{1}{2}} \\
& \leq K_{1}+K_{1}^{\frac{1}{2}} K_{2}+K_{2}^{1+\frac{1}{2}}\left\|\nabla u^{(0)}\right\|_{L^{1}(G)}^{\frac{1}{4}},
\end{aligned}
$$


which is 60 for $j=2$. Now suppose that 60 holds for some $j \geq 2$. As above for $k \in \mathbb{N}$ and for given $a_{i} \geq 0, i=1, \ldots k$, the elementary estimate

$$
\left(\sum_{i=1}^{k} a_{i}\right)^{\frac{1}{2}} \leq \sum_{i=1}^{k} a_{i}^{\frac{1}{2}}
$$

is used and again with the help of (59) we obtain

$$
\begin{aligned}
& \left\|\nabla u^{(j+1)}\right\|_{L^{1}(G)}=\left\|\nabla T\left(u^{(j)}\right)\right\|_{L^{1}(G)} \leq K_{1}+K_{2}\left\|\nabla u^{(j)}\right\|_{L^{1}(G)}^{\frac{1}{2}} \\
& \leq K_{1}+K_{2}\left(\sum_{l=1}^{j} K_{1}^{\frac{1}{2^{l-1}}} K_{2}^{\sum_{i=0}^{l-2} \frac{1}{2^{i}}}+K_{2}^{\sum_{i=0}^{j-1} \frac{1}{2^{i}}}\left\|\nabla u^{(0)}\right\|_{L^{1}(G)}^{\frac{1}{2^{j}}}\right)^{\frac{1}{2}} \\
& \leq K_{1}+K_{2}\left(\sum_{l=1}^{j} K_{1}^{\frac{1}{2^{l}}} K_{2}^{\sum_{i=0}^{l-2} \frac{1}{2^{i+1}}}+K_{2}^{\sum_{i=0}^{j-1} \frac{1}{2^{i+1}}}\left\|\nabla u^{(0)}\right\|_{L^{1}(G)}^{\frac{1}{2^{j+1}}}\right) \\
& =K_{1}+\sum_{\tilde{l}=2}^{j+1} K_{1}^{\frac{1}{2^{i-1}}} K_{2} K_{2}^{\sum_{i=0}^{\tilde{l}-3} \frac{1}{2^{i+1}}}+K_{2} K_{2}^{\sum_{i=0}^{j-1} \frac{1}{2^{i+1}}}\left\|\nabla u^{(0)}\right\|_{L^{1}(G)}^{\frac{1}{2^{j+1}}} \\
& =K_{1}+\sum_{l=2}^{j+1} K_{1}^{\frac{1}{2^{l-1}}} K_{2}^{1+\sum_{i=1}^{l-2} \frac{1}{2^{i}}}+K_{2}^{1+\sum_{i=1}^{j} \frac{1}{2^{i}}}\left\|\nabla u^{(0)}\right\|_{L^{1}(G)}^{\frac{1}{2^{j+1}}} \\
& \quad \sum_{l=1}^{j+1} K_{1}^{\frac{1}{2^{l-1}}} K_{2}^{\sum_{i=0}^{l-2} \frac{1}{2^{i}}}+K_{2}^{\sum_{i=0}^{j} \frac{1}{2^{i}}}\left\|\nabla u^{(0)}\right\|_{L^{1}(G)}^{\frac{1}{2^{j+1}}} \cdot \\
& \quad=
\end{aligned}
$$

Corollary 4.2. Let us suppose that in addition to the assumptions of Theorem 4.1 we have $K_{1}<1$, where $K_{1}$ is the constant defined in (57).

Moreover, fix $\rho>0$ and suppose that $\mathcal{W}$ is a subset of $W^{1,1}(G)$ such that for all $v \in \mathcal{W}$ we have $v=f$ on $\partial K$ and

$$
\|v\|_{W^{1,1}(G)} \leq \rho .
$$

i) Then there is a uniform constant $c=c(\rho)$ such that for all $v^{(0)} \in \mathcal{W}$, $v=v^{(n)}=T\left(v^{(n-1)}\right), n \in \mathbb{N}$, we have

$$
\|v\|_{W^{1,1}(G)} \leq c
$$

ii) For a universal constant $c$ not depending on $\rho$ we have i) for any $n \geq$ $n_{0}=n_{0}(\rho)$.

Proof. Once again recalling (10), the proof follows from Theorem 4.1.

Remark 4.1. As it should be expected, the smallness of $K_{1}$ corresponds to $c_{2} \approx c_{1}$ or $\|\nabla f\|$ small or $|G|$ small or $\lambda$ large. 


\subsubsection{Iterated fixed points on a priori sets}

In this subsection we derive the existence of iterated fixed points in a priori local bounded subsets of $W^{1,1}$.

In order to find a suitable domain $\mathcal{M}$ s.t. $T: \mathcal{M} \rightarrow \mathcal{M}$, we first let for any fixed $w \in W^{1,1}(G)$

$$
\begin{aligned}
\mathcal{T}[w] & :=\bigcup_{n=1}^{\infty} T^{n}(w) \\
& =\left\{v \in W^{1,1}(G): v=T^{n}(w) \text { for some } n \in \mathbb{N}\right\} .
\end{aligned}
$$

We then fix some $\mathcal{U} \subset W^{1,1}(G)$, where for our purposes we may suppose in the following that $v=f$ on $\partial K$ for any $v \in \mathcal{U}$.

Now let

$$
\mathcal{M}:=\mathcal{U} \cup \bigcup_{v \in \mathcal{U}} \mathcal{T}[v]=\mathcal{U} \cup T(\mathcal{U}) \cup T(T(\mathcal{U})) \ldots
$$

Clearly, if $v \in \mathcal{M}$ then $v \in \mathcal{U}$ or $v=T^{n}(w)$ for some $w \in \mathcal{U}$ and some $n \in \mathbb{N}$, hence

$$
T(v) \in \mathcal{T}[v] \cup \mathcal{T}[w] \subset \mathcal{M}
$$

With the additional notation

$$
\begin{aligned}
\mathcal{T}_{n_{0}}[w] & :=\bigcup_{k=n_{0}}^{\infty} T^{k}(w), \quad n_{0} \in \mathbb{N} \text { fixed } \\
\mathcal{M}_{n_{0}} & :=\mathcal{U} \cup \bigcup_{v \in \mathcal{U}} \mathcal{T}_{n_{0}}[v]
\end{aligned}
$$

the same reasoning as above shows for all $n \in \mathbb{N}$

$$
T^{n}: \quad \mathcal{M}_{n} \rightarrow \bigcup_{v \in \mathcal{U}} \mathcal{T}_{n}[v] \subset \mathcal{M}_{n}
$$

Increasing $\rho$ in Corollary 4.2 , $i i$ ), if necessary, and choosing $n_{0}$ sufficiently large, we now choose

$$
\mathcal{U}=\left\{v \in W^{1,1}(G):\|\nabla v\|_{L^{1}(G)} \leq \rho, v=f \text { on } \partial K\right\}
$$

such that for all $n \geq n_{0}$

$$
\bigcup_{v \in \mathcal{U}} \mathcal{T}_{n}[v] \subset \mathcal{U}=\mathcal{M}_{n} .
$$

Since $\mathcal{U}$ is a closed convex subset of $W^{1,1}(G)$, the above equality shows the same for $\mathcal{M}_{n}$, and Corollary 11.2 of [5] provides for all $n \geq n_{0}$ at least one fixed point of $T^{n}$ in $\mathcal{M}_{n}$, hence in $\mathcal{M}_{n_{0}}$. 


\subsubsection{Error estimates}

The numerical discussion of iterated fixed points is motivated by letting for all $n, k \in \mathbb{N}$ and for a given $u^{(0)}$ as above (recall (44)

$$
u^{(n+k)}=T^{n} T^{k}\left(u^{(0)}\right)=T^{n}\left(u^{(k)}\right) .
$$

With the notation

$$
R(n, k):=u^{(n+k)}-u^{(k)}
$$

we immediately obtain the first observation:

$$
T^{n}\left(u^{(k)}\right)=u^{(k)}+R(n, k),
$$

i.e. $\|R(n, k)\|$ provides a measure for the failure of $u^{(k)}$ to be numerically detected as one possible fixed point w.r.t. $T^{n}$.

Of course it is possible to choose subsequences $\left\{n_{j}\right\}$ and $\left\{k_{j}\right\}$ such that as $j \rightarrow \infty$ (recall Theorem 4.1)

$$
\left\|R\left(n_{j}, k_{j}\right)\right\|_{W^{1,1}(G)} \rightarrow 0 .
$$

These simple observations lead to the analysis of the operator $T^{n}$. In fact, if $w_{n}$ is a fixed point w.r.t. $T^{n}$,

$$
w_{n}=T^{n}\left(w_{n}\right),
$$

then we have

$$
\begin{aligned}
T\left(w_{n}\right) & =T^{n+1}\left(w_{n}\right)=T^{n}\left(w_{n}\right)+\left(T^{n+1}-T^{n}\right)\left(w_{n}\right) \\
& =w_{n}+T^{n}(T-\mathrm{id})\left(w_{n}\right) .
\end{aligned}
$$

Now a given fixed point $w_{n}$ of $T^{n}$ in general is not simultaneously a fixed point of $T$, although the converse trivially is true. The error $v_{n}:=(T-i d)\left(w_{n}\right)$ evidently has to enter the left hand side of (61).

Note that we then apply the iterated operator $T^{n}$ to $v_{n}$. This is crucial for the numerical interpretation: Decreasing $\sigma$ in the kernel appearing in $T=T$ means to blow up the error via the non-contracting operator $T$ in $T^{n}$ which exactly describes the behavior of the examples sketched in the introduction.

\section{Acknowledgement}

The research of M.C. and J.W. has received funding by the European Research Council (ERC) under the European Union's Horizon 2020 research and innovation programme (grant agreement no. 741215, ERC Advanced Grant INCOVID). 


\section{References}

[1] Adams, R.A.: Sobolev Spaces. Academic Press, London (1975)

[2] Catté, F., Lions, P.L., Morel, J.M., Coll, T.: Image selective smoothing and edge detection by nonlinear diffusion. SIAM Journal on Numerical Analysis 32, 1895-1909 (1992)

[3] Charbonnier, P., Blanc-Féraud, L., Aubert, G., Barlaud, M.: Two deterministic half-quadratic regularization algorithms for computed imaging. In: Proc. 1994 IEEE International Conference on Image Processing. vol. 2, pp. 168-172. IEEE Computer Society Press, Austin, TX (Nov 1994)

[4] Galić, I., Weickert, J., Welk, M., Bruhn, A., Belyaev, A., Seidel, H.P.: Image compression with anisotropic diffusion. Journal of Mathematical Imaging and Vision 31(2-3), 255-269 (Jul 2008)

[5] Gilbarg, D., Trudinger, N.S.: Elliptic Partial Differential Equations of Second Order. Springer, Berlin, revised third printing, second edn. (1998)

[6] Ladyzhenskaya, O.A., Uraltseva, N.N.: Linear and Quasilinear Elliptic Equations. Academic Press, New York (1968)

[7] Mainberger, M., Hoffmann, S., Weickert, J., Tang, C.H., Johannsen, D., Neumann, F., Doerr, B.: Optimising spatial and tonal data for homogeneous diffusion inpainting. In: Bruckstein, A.M., ter Haar Romeny, B., Bronstein, A.M., Bronstein, M.M. (eds.) Scale Space and Variational Methods in Computer Vision, Lecture Notes in Computer Science, vol. 6667, pp. 26-37. Springer, Berlin (2012)

[8] Morrey, C.B.: Multiple Integrals in the Calculus of Variations. Springer, Berlin (1966)

[9] Perona, P., Malik, J.: Scale space and edge detection using anisotropic diffusion. IEEE Transactions on Pattern Analysis and Machine Intelligence 12, 629-639 (1990)

[10] Schmaltz, C., Peter, P., Mainberger, M., Ebel, F., Weickert, J., Bruhn, A.: Understanding, optimising, and extending data compression with anisotropic diffusion. International Journal of Computer Vision 108(3), 222-240 (Jul 2014)

[11] Weickert, J.: Theoretical foundations of anisotropic diffusion in image processing. Computing Supplement 11, 221-236 (1996) 
[12] Weickert, J.: Anisotropic Diffusion in Image Processing. Teubner, Stuttgart (1998)

[13] Weickert, J., Welk, M.: Tensor field interpolation with PDEs. In: Weickert, J., Hagen, H. (eds.) Visualization and Processing of Tensor Fields, pp. 315-325. Springer, Berlin (2006)

Michael Bildhauer* bibi@math.uni-sb.de

Marcelo Cárdenas** cardenas@mia.uni-saarland.de

Martin Fuchs* fuchs@math.uni-sb.de

Joachim Weickert** weickert@mia.uni-saarland.de

* Department of Mathematics

Saarland University

Faculty Math. and Computer Sci.

P.O. Box 151150

66041 Saarbrücken, Germany
** Math. Image Analysis Group

Saarland University

Faculty Math. and Computer Sci.

Campus E1.7

66041 Saarbrücken, Germany 